\title{
"Pine Mouth" Syndrome: Cacogeusia Following Ingestion of Pine Nuts (Genus: Pinus). An Emerging Problem?
}

\author{
Marc-David Munk
}

Published online: 5 January 2010

(C) American College of Medical Toxicology 2009

\begin{abstract}
We report a case of cacogeusia, specifically metallogeusia (a perceived metallic or bitter taste) following pine nut ingestion. A 36-year-old male presented with cacogeusia one day following ingestion of 10-15 roasted pine nuts (genus: Pinus). Symptoms became worst on postexposure day 2 and progressively improved without treatment over 5 days. There were no other symptoms and physical examination was unrevealing. All symptoms resolved without sequalae. We contemporaneously report a rise in pine nut-associated cacogeusia reported online during the first quarter of 2009, and a significant rise in online searches related to pine nut-associated cacogeusia (or what the online public has termed "pine mouth") during this time. Most online contributors note a similar cacogeusia 1-3 days following pine nut ingestion lasting for up to 2 weeks. All cases seem self-limited. Patients occasionally describe abdominal cramping and nausea after eating the nuts. Raw, cooked, and processed nuts (in pesto, for example) are implicated. While there appears to be an association between pine nut ingestion and cacogeusia, little is known about this condition, nor can any specific mechanism of specific cause be identified. It is not known if a specific species of pine nut can be implicated. "Pine mouth" appears to be an emerging problem.
\end{abstract}

Keywords Cacogeusia $\cdot$ Dysgeusia $\cdot$ Metallogeusia $\cdot$ Pinus

No funding was received

M.-D. Munk $(\bowtie)$

Department of Emergency Medicine, University of New Mexico, MSC 10 5560, 1 University of New Mexico,

Albuquerque, NM 87131-0001, USA

e-mail: MMunk@salud.unm.edu

\section{Introduction}

Cacogeusia may have various causes. These include metal toxicity, seafood toxins, nutritional disorders (especially zinc deficiency), and neurologic disorders [1]. Medications, particularly angiotensin-converting enzyme inhibitors, disulfiram, calcium channel blockers, and diuetics can cause cacogeusia by interfering with taste receptors, or with receptor mechanisms [2-4]. Dental conditions and infections such as sinusitis may also cause symptoms. We report a case of cacogeusia, specifically metallogeusia (a perceived metallic or bitter taste) following pine nut ingestion and suggest a possible association between pine nut ingestion and subsequent prolonged cacogeusia.

\section{Case Report}

A 36-year-old, previously well male physician, presented to colleagues with pronounced bitter and metallic taste, exacerbated by ingestion of sweet foods and drinks. Symptoms began $24 \mathrm{~h}$ following a large meal containing well-cooked meat, vegetables, salad, red wine, and pine nuts that had been lightly roasted. Approximately $10-15$ pine nuts were ingested. No fish or shellfish had been ingested during the previous 2 weeks. There were no other complaints, including abdominal discomfort, nausea, vomiting or diarrhea, and no rash was noted. No other neurologic complains were noted including cacosmia. Social history was unrevealing: the patient was a non-smoker, drank three to four alcoholic beverages weekly and denied any history of illicit substance use. An otherwise healthy physician, he denied any use of either prescribed or over-the-counter medications, or nutritional supplements/ herbal preparations, during the 2 weeks prior to symptom onset. Physical examination was unrevealing: the patient was 
afebrile and vital signs were normal for age. Neurologic testing (including cranial nerves II-XII; motor, sensory, and reflex testing; gait and coordination) was normal. Dermatologic examination showed no lesions. A normal complete dental examination had been performed 1 week prior to symptoms, and examination of the oropharynx after symptoms began revealed no observable pathology. Ineffective therapies attempted during this episode included mouthwash gargles and saline gargles. Cacogeusia was worst on postexposure day 2 , progressively improving without treatment over 5 days. No laboratory testing was performed, and symptoms resolved without sequalae.

\section{Discussion}

Cacogeusia following pine nut ingestion appears to have been first described at a European medical conference in 2001 [5]. Authors hypothesized that oxidized pine nuts were responsible for eight cases of bitter taste disturbance, six which had been reported to a European poison control center. They were able to elicit cacogeusia in a healthy physician volunteer who ingested implicated pine nuts. Chromatographic examination of the suspicious pine nuts revealed novel triglycerides, potentially created by oxidative stresses, as a possible cause. A specific receptor or mechanism was not offered.

There were few reported subsequent cases of pine nutassociated cacogeusia between 2001 and 2009. In the second quarter of 2009 , following our index case, we noted a spike in cases of pine nut-associated cacogeusia reported online [6-9] and in the mainstream media [10]. Termed "Pine Mouth" by the public, cases present in a roughly similar fashion: a persistent metallic or bitter taste beginning 1-3 days following ingestion of pine nuts lasting for up to 2 weeks. All cases seem self-limited. Patients occasionally describe abdominal cramping and nausea after eating the nuts. Raw, cooked, and processed nuts (in pesto, for example) have been implicated. Online reports implicated pine nuts purchased in supermarkets, restaurants, and bulk food warehouses in the US and in the UK.

To quantify this increase in interest in pine nut-associated cacogeusia, we searched Google Trends (which reports search word volume indices over time) for the terms "Pine Mouth" or "Pine Bitter" or "Pine Taste." This technique of using Google search volume as a barometer of disease prevalence has been previously used in influenza epidemiology. In that setting, search volume was found to correlate well with physician visits for flu-like symptoms within defined geographic areas and searches were found to estimate flu activity in near realtime $[11,12]$. We surmised that this informal surrogate search method would provide a rough measure of public interest in "pine mouth." We found, for all three searchterms, a rapid spike (search volume indices 100-150 times baseline) in search popularity abruptly beginning in the second quarter of 2009 [13]. Whether this reflects a surge in cases, or public interest due to media reports, is not known.

This case report raises an intriguing hypothesis, but by no means proves causality. Little is known about the cacogeusia termed "pine mouth". Initial reports suggested that affected nuts came from China, although consumer reports online show that symptom-causing pine nuts originated from other areas as well. It is unclear if a specific species of Pinus is to blame. If a link between pine nut ingestion and cacogeusia is real, an exact mechanism is unclear: symptoms may be related to decomposing lipids, as pinus oils are known to be unstable and degrade quickly [14]. The role of toxins and other contaminants has not been fully evaluated. "Pine Mouth" appears to be an emerging problem-bothersome, but apparently self-limited and relatively benign.

Conflicts of Interest None declared.

\section{References}

1. Heckmann JG, Lang CJ (2006) Neurological causes of taste disorders. Adv Otorhinolaryngol 63:255-264

2. Chiang WK (2006) Otolaryngologic principles. In: Flomenbaum NE, Goldfrank LR, Hoffman RS, Howland MA, Lewin NA, Nelson LS (eds) Goldfrank's toxicologic emergencies, 8th edn. McGraw-Hill, New York

3. Ackerman BH, Kasbekar N (1997) Disturbances of taste and smell induced by drugs. Pharmacotherapy 17(3):482-496

4. Henkin RI (1994) Drug-induced taste and smell disorders. Incidence, mechanisms and management related primarily to treatment of sensory receptor dysfunction. Drug Saf 11(5):318-377

5. Mostin M (2001) Taste disturbances after pine nut ingestion (abstract). Eur J Emerg Med 8(1):76

6. The last word: transient taste. 2008 [updated 2008]. Available from http://www.newscientist.com/blog/lastword/2008/08/tran sient-taste.html. Accessed 18 May 2009

7. Bitter taste in mouth while eating. 2008 [updated 2008] Available from http://www.steadyhealth.com/Bitter_taste_in_mouth_while eating-t85978-0-asc-90.html. Accessed 18 May 2009

8. The great pine nut mystery. 2009 [updated 2009]. Available from: http://lukehoney.typepad.com/the_greasy_spoon/2009/02/thegreat-pine-nut-mystery.html. Accessed 18 May 2009

9. Pine nuts left a bitter taste in my mouth. 2009 [updated 2009]. Available from http://pinchmysalt.com/2009/05/02/pine-nuts-lefta-bitter-taste-in-my-mouth/. Accessed 18 May 2009

10. Middleton C. Pine mouth puzzle: why do these nuts leave you with a bitter taste? The Daily Mail. 18 May 2009

11. Ginsberg J, Mohebbi MH, Patel RS, Brammer L, Smolinski MS, Brilliant L (2009) Detecting influenza epidemics using search engine query data [letter]. Nature 457:1012-1014

12. Google.org. Flu Trends. How does this work? 2009 [updated 2009]. Available from http://www.google.org/about/flutrends/how. html. Accessed 18 June 2009

13. Google. About Google Trends. 2009 [updated 2009]. Available from http://www.google.com/intl/en/trends/about.html. Accessed 18 June 2009

14. Miraliakbari H, Shahidi F (2008) Oxidative stability of tree nut oils. J Agric Food Chem 56(12):4751-4759 